\title{
PLANAR AND STRONGLY UNIFORM NEAR-RINGS ${ }^{1}$
}

\author{
GEORGE SZETO
}

\begin{abstract}
The concept of a finite strongly uniform near-ring defined by $\mathbf{G}$. Ferrero is generalized to the infinite case. A relation between planar near-rings and strongly uniform near-rings is studied. A structure theorem for an integral planar near-ring of M. Anshel and J. Clay is extended to a strongly uniform nearring.
\end{abstract}

1. Introduction. Let $R$ be a near-field as defined in [8] and [9]. The additive group of $R$ was shown to be commutative in [8]. Recently, more works have been done on such an $R$ and on other more general algebraic systems. Some geometric interpretations were found and some BIBD (balanced incomplete block designs) were constructed by using these syștems. Moreover, Anshel and J. Clay generalized the concept of planarity in [9] and found more properties of a planar near-ring [1], [2] and [3]. Also, Ferrero studied a more general system than planar near-rings called strongly uniform near-rings [4] and [5]. In [5] he gave a necessary and sufficient condition for a strongly uniform near-ring being a planar near-ring as follows: A finite near-ring is a planar near-ring if and only if it is strongly uniform, the multiplication is nontrivial and all nonzero orbits under the automorphism group induced by the elements of the nearring are principal [5, Theorem 1]. The purpose of the present paper is to generalize the above theorem of Ferrero to an infinite case. It is proved that the necessity still holds while the sufficiency does not. Moreover, an elementary proof of the sufficient condition of the Ferrero theorem is given. Finally, some classes of infinite strongly uniform near-rings are obtained and the structural Theorem 1 in [1] is also extended. Our techniques are a combination of [1], [5] and [9].

2. Basic definitions and notations. A (left) near-ring $R$ is an algebraic system $(R,+, \cdot)$ such that $(1)(R,+)$ is a group (not necessarily commutative); (2) $(R, \cdot)$ is a semigroup; (3) multiplication - is left distributive over addition + . Examples can be found in references.

Received by the editors January 3, 1972.

AMS (MOS) subject classifications (1970). Primary 16A76; Secondary 16A78.

Key words and phrases. Equivalent multipliers, near-rings, integral planar near-rings, planar near-rings, strongly uniform near-rings.

${ }^{1}$ This work was done under the support of NSF Grant 3320.

(c) American Mathematical Society 1974 
Let $a$ and $b$ be elements in $R$ such that $a c=b c$ for all $c$ in $R$. Then $a$ and $b$ are called left equivalent multipliers denoted by $a \equiv_{m} b[3$, p. 319]. We know that the relation $\equiv_{m}$ is an equivalence relation on $R$.

If the equation, $a x=b x+c$, has a unique solution $x$ in $R$ for $a, b$ and $c$ in $R$ with $a \not_{m} b$ then we call $R$ a near-ring with planar property [3, p. 319].

$R$ is called a planar near-ring if (1) $R$ has planar property, and (2) there are at least three equivalence classes with respect to $\equiv_{m}$ in $R[3, \mathrm{p} .319]$.

Throughout, $R$ is assumed to be a (left) near-ring. The following identities will be used freely: $r 0=0$ and $r(-s)=-r s$ for all $r, s$ in $R$, where 0 is the identity of $(R,+)$.

3. Strongly uniform and planar near-rings. Ferrero called a near-ring $R$ strongly uniform if there is at least one nonzero divisor in $R$ and if the right annihilator of each element $r$ in $R$ is either 0 or $R$; that is, $A(r)=$ $\{s / r s=0\}$ is either 0 or $R$ [4, Definition III]. The multiplication of $R$ is called trivial if for each $a$ in $R$ either $a R=0$ or $a b=b$ for all $b$ in $R$. Obviously, for each $r$ with $A(r)=0$, the map $\varphi_{r}: x \rightarrow r x$ is an automorphism of $(R,+)$ in case $R$ is finite. We shall keep the same definition of a strongly uniform near-ring as defined by Ferrero even in the infinite case. First, let us recall a theorem of Ferrero:

THEOREM 3.1. A finite near-ring $R$ is a planar near-ring if and only if it is strongly uniform, the multiplication of $R$ is nontrivial and all nonzero orbits of $(R,+)$ under the automorphism group $\Phi$ induced by the elements of $R$ are principal; that is, for each $r^{\prime}, r^{\prime \prime}$ in the same nonzero orbit, there is only one $\varphi_{s} \in \Phi$ such that $\varphi_{s}\left(r^{\prime}\right)=r^{\prime \prime}[5$, Theorem 1].

In this section, we shall prove the necessity and disprove the sufficiency of the above theorem in the infinite case. First, by the same proof as given in $[1$, p. 167], we have:

LEMMA 3.2. If $R$ is a planar near-ring then it is strongly uniform such that $A(0)=R$.

Lemma 3.3. If $R$ is a planar near-ring, then (1) every element $r$ with $A(r)=0$ induces a group automorphism $\varphi_{r}$ of $(R,+)$ defined by $\varphi_{r}(s)=r s$ for all $\sin R$, and (2) the set $\Phi=\left\{\varphi_{r} / A(r)=0\right\}$ forms an automorphism group of $(R,+)$ under composition operation.

PRoof. (1) Since $r(x+y)=r x+r y, \quad \varphi_{r}(x+y)=\varphi_{r}(x)+\varphi_{r}(y)$ for all $x$ and $y$ in $R$. Hence $\varphi_{r}$ is a homomorphism of $(R,+)$. Moreover, let $\varphi_{r}(x)=0, r x=0 . A(r)=0$, so $x=0$, and so $\varphi_{r}$ is one-to-one. Furthermore, by Lemma 3.2, $A(0)=R$. But $A(r)=0$, then $0 \not \equiv_{m} r$. Thus the equation, 
$r x=0 x+c$, has a unique solution $x$ in $R$ for an arbitrary $c$ in $R$; that is, $r x=c ; \varphi_{r}(x)=c$ has a unique solution $x$ in $R$. Therefore $\varphi_{r}$ is onto. Consequently, it is an automorphism of $(R,+)$.

(2) It is clear that the set $\Phi=\left\{\varphi_{r} / A(r)=0\right\}$ is closed and associative under composition operation. Next, by Lemma 3.2 again, $r \equiv_{m} 0$, so $r x=0 x+r$ has a unique solution $1_{r}$; and so $r 1_{r}=r$. But then $r 1_{r}^{2}=r 1_{r}$ and $r\left(1_{r}^{2}-1_{r}\right)=0$. Noting $A(r)=0$ we have $1_{r}^{2}=1_{r}$. Thus $1_{r}$ is a nonzero idempotent. Obviously, $A\left(1_{r}\right)=0$, so $\varphi_{1_{r}}$ is in $\Phi$. On the other hand, the Pierce decomposition theorem holds for near-rings, so $R=1_{r} R+R^{\prime}$ with $1_{r} R^{\prime}=0$. But then $A\left(1_{r}\right)=0$ implies $R^{\prime}=0$. Therefore, $R=1_{r} R$. This implies that $1_{r}$ is a left identity of $R$; and hence $\varphi_{1_{r}}$ is the identity of $\Phi$. Finally, we claim that every $\varphi_{r}$ in $\Phi$ has an inverse. In fact, the equation, $r x=0 x+1_{r}$, has a unique solution $r^{\prime}$ such that $r r^{\prime}=1_{r}$. Obviously, $A\left(r^{\prime}\right)=0$, but then $\varphi_{r} \varphi_{r^{\prime}}=\varphi_{r r^{\prime}}=\varphi_{1_{r}}$. The proof is thus completed.

Now we show:

THEOREM 3.4. Let $R$ be a planar near-ring (finite or infinite). Then it is strongly uniform, the multiplication of $R$ is nontrivial and all nonzero orbits of $(R,+)$ under the automorphism group induced by the elements of $R$ are principal.

Proof. Since $R$ is a planar near-ring, there are at least three equivalence classes of left equivalent multipliers and hence the multiplication of $R$ is nontrivial. Moreover, by Lemma 3.2, $R$ is strongly uniform, so it suffices to show that all nonzero orbits of $(R,+)$ under $\Phi$ are principal. Let $a$ and $b$ be two elements in a nonzero orbit. Suppose there were two different automorphisms $\varphi_{r}$ and $\varphi_{r^{\prime}}$ in $\Phi$ such that $\varphi_{r}(a)=\varphi_{r^{\prime}}(a)=b$. Then $r a=r^{\prime} a$. But $\varphi_{r} \neq \varphi_{r^{\prime}}$ implies $r \not \equiv_{m} r^{\prime}$; then the equation, $r x=r^{\prime} x+0$, has a unique solution. Noting that $x=0$ and $x=a$ are solutions, we conclude that $a=0$. This is a contradiction. Therefore there is a unique $\varphi_{r}$ such that $\varphi_{r}(a)=b$. The proof is thus completed.

Next, the sufficiency is disproved by a counter example. Let $Z$ be the ring of integers and let $Z \times Z=\{(n, m) / n$ and $m \in Z\}$ be the direct sum of two copies of $(Z,+)$. Also, it is a $Z$-module by defining $k(n, m)=(k n, k m)$ for all $k$ in $Z$. Then a multiplication can be defined so that $(Z \times Z,+, *)$ is a near-ring; that is, $\left(n^{\prime}, m^{\prime}\right) *\left(n^{\prime \prime}, m^{\prime \prime}\right)=n^{\prime}\left(n^{\prime \prime}, m^{\prime \prime}\right)$. It is easy to show that $(1)(Z \times Z)$ is an infinite near-ring; (2) it is strongly uniform with an automorphism group $\Phi$ induced by the elements of $R$ isomorphic with $(Z /(2),+)$; (3) the multiplication is nontrivial; and (4) all nonzero orbits under $\Phi$ are principal. On the other hand, it can be shown to be nonplanar. In fact, the equation, $(2,0) x=(0,0) x+(1,1)$ with $(2,0) \not \equiv_{m}(0,0)$, does not have a solution. 
Here, we present an elementary proof of the sufficiency of the Ferrero theorem in the finite case.

THEOREM 3.5 (FERRERo). If $R$ is a finite near-ring such that (1) it is strongly uniform, (2) the multiplication is nontrivial, and (3) all nonzero orbits under $\Phi$ are principal, then $R$ is a planar near-ring [5, Theorem 1].

Proof. Since the multiplication of $R$ is nontrivial, there are at least three equivalence classes of left equivalent multipliers in $R$, so, it suffices to show that $R$ has planar property; that is, for $a \neq_{m} b$, the equation, $a x=b x+c$, has a unique solution. Let us consider a map $f$ from $R$ to $R$ defined by $f(r)=-b r+a r$. We claim that $f$ is one-to-one. Assume $f(r)=$ $f\left(r^{\prime}\right)$; then $-b r+a r=-b r^{\prime}+a r^{\prime}$. This implies that $b r^{\prime}-b r=a r^{\prime}-a r$, $b\left(r^{\prime}-r\right)=a\left(r^{\prime}-r\right)$. In case $A(a)=R$ and $A(b)=0, b\left(r^{\prime}-r\right)=0$; and hence $r^{\prime}-r=0, r^{\prime}=r$. The similar argument holds for the case, $A(a)=0$ and $A(b)=R$. In case $A(a)=0$ and $A(b)=0$ with $a \equiv_{m} b, \varphi_{a} \neq \varphi_{b}$ in $\Phi$ with $\varphi_{a}\left(r^{\prime}-r\right)=\varphi_{b}\left(r^{\prime}-r\right)$. Noting that $\left(r^{\prime}-r\right)$ and $\varphi_{a}\left(r^{\prime}-r\right)$ are in the same orbit, we have that $r^{\prime} \neq r$ forces $\varphi_{a}=\varphi_{b}$ because all nonzero orbits under $\Phi$ are principal. This leads to a contradiction that $a \equiv_{m} b$ (for $\varphi_{a}=\varphi_{b}$ ). Thus $r=r^{\prime}$. Therefore $f$ is one-to-one; and hence it is onto (for $R$ is finite). This implies that $f(x)=c$ has a unique solution, or $a x=b x+c$ has a unique solution $x$ in $R$.

4. A classification of strongly uniform near-rings. The near-ring $R$ in the last section was assumed to have at least three equivalence classes of left equivalent multipliers and to be strongly uniform. In this section, we shall discuss the case in which there are not more than two classes. Also, the structural theorem for an infinite (or finite) integral planar near-ring [1, Theorem 1] is extended.

THEOREM 4.1. Let $R$ be a strongly uniform near-ring. If there is only one equivalence class of left equivalent multipliers, then $a b=b$ for all $a$ and $b$ in $R$.

Proof. First, we note that $R=0 R+R^{\prime}$ with $0 R^{\prime}=0$ by the Pierce decomposition theorem. Since $R$ is strongly uniform, either $R^{\prime}=0$ or $0 R=0$. In case $R^{\prime}=0, R=0 R$. Hence $R=\{0 r / r$ in $R\}$. This implies $(0 r)\left(0 r^{\prime}\right)=0 r^{\prime}$; that is, $a b=b$ for all $a$ and $b$ in $R$. Next, in case $0 R=0$, $R=R^{\prime}$. Hence $0 r=0=a r$ for all $a$ and $r$ in $R$ because $0 \equiv_{m} a$. This contradicts that there exists at least one nonzero divisor in $R$ (for $R$ is strongly uniform). Thus the proof is completed. 
THEOREM 4.2. Let $R$ be a strongly uniform near-ring with a left identity. If there are exactly two classes of left equivalent multipliers, then $0 r=0$ for all $r$ in $R$ and $a b=b$ for all $a$ and $b$ in $R$ with $a \equiv_{m} 0$.

Proof. By the Pierce decomposition theorem again, $R=0 R+R^{\prime}$ with $0 R^{\prime}=0$. There are exactly two classes of left equivalent multipliers, so $0 R=0$; for otherwise $0 R=R$ implies that there is only one class by Theorem 4.1. On the other hand, let $e$ be a left identity of $R$ by hypothesis, then $a r=e r=r$ for all $a$ with $a \equiv_{m} e$ and $b r=0 r=0$ for all $b$ with $b \equiv_{m} 0$.

We conclude this section with extending Theorem 1 in [1].

THEOREM 4.3. Let $R$ be a strongly uniform near-ring. Assume there are at least three classes of left equivalent multipliers. If the set $\Phi=\left\{\varphi_{s} \mid r \rightarrow s r\right.$ for all $r$ in $R$, where $A(s)=0\}$ is an automorphism group of $(R,+)$ under the composition operation, then (1) the set $S=\{s \mid A(s)=0\}$ is a union of groups, $S x$, such that $S x \cap S y=\varnothing$ for different left identities $x$ and $y$ of $R$; (2) $S x \cong S y$ as a group isomorphism.

Proof. Since $\Phi$ is an automorphism group of $(R,+), s R=R$ for all $s$ in $S$. Hence there exists a $1_{s}$ such that $s 1_{s}=s$. Thus every element in $S$ has a right identity. Moreover, $A(s)=0$, so $1_{s}$ is unique. Also, noting that $1_{s}^{2}=1_{s}$ and that $A\left(1_{s}\right)=0$, we have a left identity $1_{s}$ of $R$. Furthermore, $s R=R$ implies that there exists a right inverse of $s$. It is easy to see that the right inverse of $s$ is unique. Thus the theorem can be completed by applying the arguments given in [1, Theorem 1].

REMARK. There are near-rings satisfying the conditions of the above theorem and they are not planar. For example, let $R$ be a $p$-singular and a nonsemisimple finite near-ring for a prime integer $p$. Then (1) $R$ is strongly uniform $\left[4\right.$, Theorem 5]; (2) $\Phi=\left\{\varphi_{s} \mid A(s)=0\right\}$ is an automorphism group of $(R,+)$ because $R$ is finite; (3) $\Phi$ has nonfixed point free automorphisms with points in $A=\{a \mid A(a)=R\}$ [4, Theorem 17]. Therefore, $R$ is not a planar near-ring [5, Theorem 1].

The author wishes to thank Professor Ferrero for the above example. Ferrero proved a similar result to Theorem 4.2 [5, Corollary 1].

\section{REFERENCES}

1. M. Anshel and J. Clay, Planar algebraic systems: Some geometric interpretations, J. Algebra 10 (1968), 166-173. MR 39 \#2813.

2. _ Planarity in algebraic systems, Bull. Amer. Math. Soc. 74 (1968), 746-748. MR 37 \#1415.

3. J. Clay, Generating balanced incomplete block designs from planar near-rings, J. Algebra 22 (1972), 319-331.

4. G. Ferrero, Struttura degli stems p-singolari, Riv. Mat. Univ. Parma (2) 7 (1966), 243-254. MR 37 \#4130.

5. —_ Stems planari e BIB-designi, Riv. Mat. Univ. Parma (2) 11 (1970), 79-96. 
6. G. Szeto, Finite near-rings with trivial annihilators, J. Austral. Math. Soc. (to appear).

7. - The sub-semi-groups excluding zero of a near-ring, Monatsh. Math. 77 (1973), 357-362.

8. H. Zassenhaus, Über endliche Fastkörper, Abh. Math. Sem. Univ. Hamburg 11 (1936), 187-220.

9. J. Zemmer, Near-fields, planar and non-planar, Math. Student 32 (1964), 145-150. MR 31 \#5888.

Department of Mathematics, Bradley University, Peoria, Illinois 61606 